www.jmscr.igmpublication.org

Impact Factor 5.84

Index Copernicus Value: 83.27

ISSN (e)-2347-176x ISSN (p) 2455-0450

crossref DOI: https://dx.doi.org/10.18535/jmscr/v5i3.29

Journal Of Medical Science And Clinical Research

IGM Publication

An official Publication of IGM Publication

\title{
Detection of Diffusion-Weighted and Apparent Diffusion Coefficient MRI Abnormalities in 100 Patients with Stroke
}

\author{
Authors \\ Dr Amaresh Kumar. A ${ }^{1}$, Dr Elamparidhi. $P^{2}$, Dr Ramesh Kumar. $\mathbf{R}^{\mathbf{3}}$, \\ Dr Yash Achanthani ${ }^{4}$ \\ ${ }^{1,4}$ Post Graduate Radio-Diagnosis, SMVMCH, Puducherry \\ ${ }^{2}$ Senior Resident Radio-Diagnosis, SMVMCH, Puducherry \\ ${ }^{3}$ Prof and HOD Radio-Diagnosis, SMVMCH, Puducherry
}

\begin{abstract}
Background and Purpose: Diffusion-weighted MRI (DWI) is highly sensitive in detecting early cerebral ischemic changes in acute stroke patients. In this study we compared the sensitivity of DWI with that of conventional MRI techniques.

Materials and Methods: We performed DWI, ADC, fluid-attenuated inversion recovery, spin-echo T2 and T1 weighted MRI sequences in 100 patients with suspected stroke. Imaging features of DWI, ADC were correlated with the routine MRI sequences.

Results: The study comprised $61 \%$ males and 39\% females. Infarcts constituted $100.0 \%$ of cases of the total cases in this study. Of this, $72.0 \%$ were acute infarcts, $22.0 \%$ were chronic infarcts and $6.0 \%$ were sub-acute infarcts. All cases of acute infarcts and 33\% of sub-acute infarcts showed diffusion restriction. None of the chronic infarcts showed true restriction of diffusion. No signal abnormality was noted in $13.8 \%$ of acute infarcts on $T 2 W$ images.

Conclusions: Diffusion weighted MRI has been proven to be of excellent use in the characterization of infarcts and in the detection of acute infarcts. It is especially useful in the initial few hours of the ischemic insult when conventional MR sequences may be inconclusive and may not detect the infarct. In the setting of multiple infarcts it helps detect the acute ones and is helpful in differentiating acute, sub-acute and chronic infarcts. Thus diffusion weighted MR imaging has to be included in any standard imaging protocol for stroke patients.

Key Words: cerebral infarction, magnetic resonance imaging, DWI, ADC, acute stroke, chronic stroke, gliosis.
\end{abstract}

\section{INTRODUCTION}

Diffusion-weighted imaging is an MR imaging technique in which contrast within the image is based on microscopic motion of water. Diffusionweighted images are obtained by adding a series of two sequential gradient pulses to a 90-180degree spin-echo sequence.
A value known as apparent diffusion coefficient (ADC) is determined by diffusion weighting of the imaging sequence. This value is dependent on a number of variables including time, orientation of the imaging plane, the tissue being imaged, and the energy state of the imaged tissue. Signal intensity on a gray scale is directly related to ADC 
values on DWI. Brain tissue with low ADC values appears relatively hypointense, whereas regions with higher ADC values appear hyper intense.

Diffusion weighted imaging is a technique that assesses local environment at the cellular level to determine changes in the random movement of water protons. Restricted diffusion appears as an area of increased signal on DWI and reduced signal on ADC maps.

Whereas DWI is most often used to identify acute arterial ischemia, other processes that interfere with or restrict the movement of water can cause notable changes on DWI.

DWI images aid in the diagnosis of various intracranial lesions and by comparing with the ADC and FLAIR images it will also be possible to characterise these lesions. DWI also helps to interpret the age of a lesion as in infarct and haemorrhage.

\section{AIMS AND OBJECTIVES}

1. To describe the usefulness of Diffusion Weighted MRI in imaging various stroke patients.

2. To discuss the imaging features in DWI and ADC of various arterial ischemia.

This study is descriptive study based on data collected from January 2016 to August2016 in the Department of Radiodiagnosis, Sri Manakula Vinayagar Medical College and Hospital, Puducherry.

\section{SOURCE OF DATA:}

The source of data for this study is patients referred to the Department of Radiodiagnosis, Sri Manakula Vinayagar Medical College and Hospital, Puducherry, for MRI brain with diffusion weighted imaging. This consists of a study of 50 patients with arterial ischemia detected on imaging.

The MRI was done on the advice of the referring doctor and no patient was made to undergo MRI for the sole purpose of this study.

\section{Inclusion Criteria}

The criteria for inclusion of the patients in the study included those patients who were clinically referred for diffusion weighted MRI of the brain and were detected to have Infarction and hypoxic ischemic injury

\section{Exclusion Criteria}

1. Infarcts with hemorrhagic transformation.

2. Intra-axial / extra-axial tumours

3. Vascular malformations such as AVM, Cavernous angiomas,etc.

4. Congenital malformations of the central nervous system.

5. Contraindications for MRI: claustrophobia, cochlear implant, etc.

\section{Demographic Characteristics}

1. Type of study: descriptive

2. Age: all age groups

3. Sex: both male and female

\section{Data Acquisition}

Patients referred for diffusion weighted MRI of the brain, underwent the examinationafter contraindications for MRI were excluded and consent was taken.

All the MRI scans in this study were performed using 1.5 T MRI scanner (Philips)

\section{Imaging Methods}

- Equipment: 1.5 Tesla (Philips)

- All patients were examined in supine position.

- $\quad$ Sagittal T1W sequence.

- Axial T1W and T2W sequences.

- Slice thickness of 3-4mm with $1 \mathrm{~mm}$ gap, a 192 x 256 acquisition matrix and one excitation.

- For T1W spin-echo sequences: TR of 500$600 \mathrm{~ms}$ and TE of $20 \mathrm{~ms}$ or less.

- For T2W spin-echo sequences: TR of 2500 to $3000 \mathrm{~ms}$ and $\mathrm{TE}$ of 30 to $60 \mathrm{~ms}$ (first echo) and 70 to $120 \mathrm{~ms}$ (second echo).

- Axial FLAIR (Fluid Attenuation Inversion Recovery) sequence (TR of $10000 \mathrm{~ms}$, TE of $100 \mathrm{~ms}$ and TI of $2200 \mathrm{~ms}$ ).

- Axial DWI and ADC 


\section{JMSCR Vol||05||Issue||03||Page 18450-18456||March}

The present study was carried out to describe imaging characteristics of intracranial lesions on DWI and to compare them with ADC and T2 FLAIR images.

Findings in the patients studied were tabulated using Microsoft Excel.

All statistical analyses were conducted using the SPSS statistical package (version 16.0).

50 cases of arterial ischemia were included in the study. The observations of these 50 patients were compiled and analyzed.

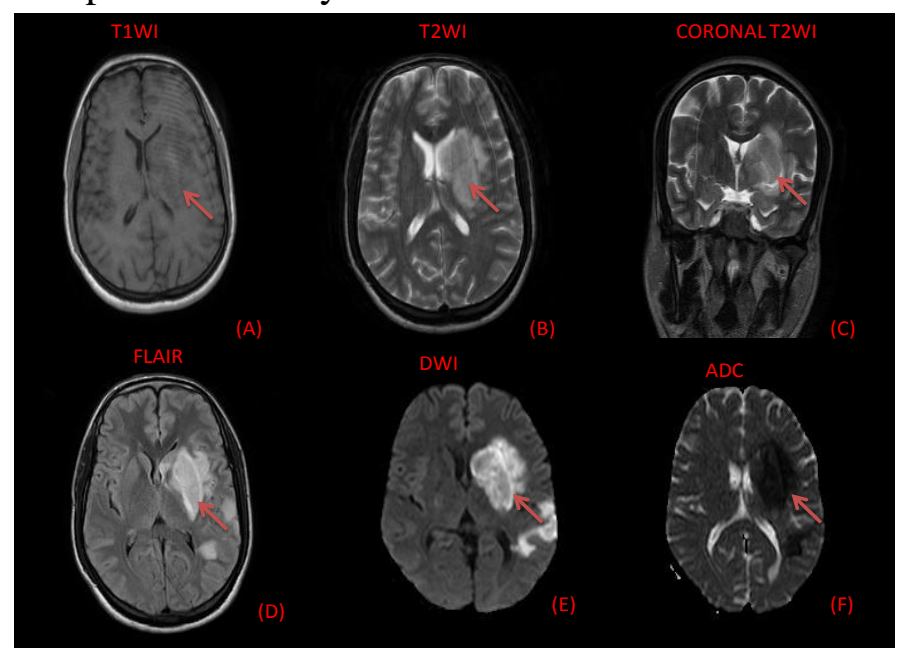

Figure 1: MRI images of an acute left MCA territory infarct. T1WI axial sequence shows hypointense area in the left gangliocapsular region (A), T2WI axial and coronal and FLAIR sequences shows hyperintensity (B, C, D), Diffusion restriction is noted in the DWI sequence (E) and ADC shows hypointensity (F)

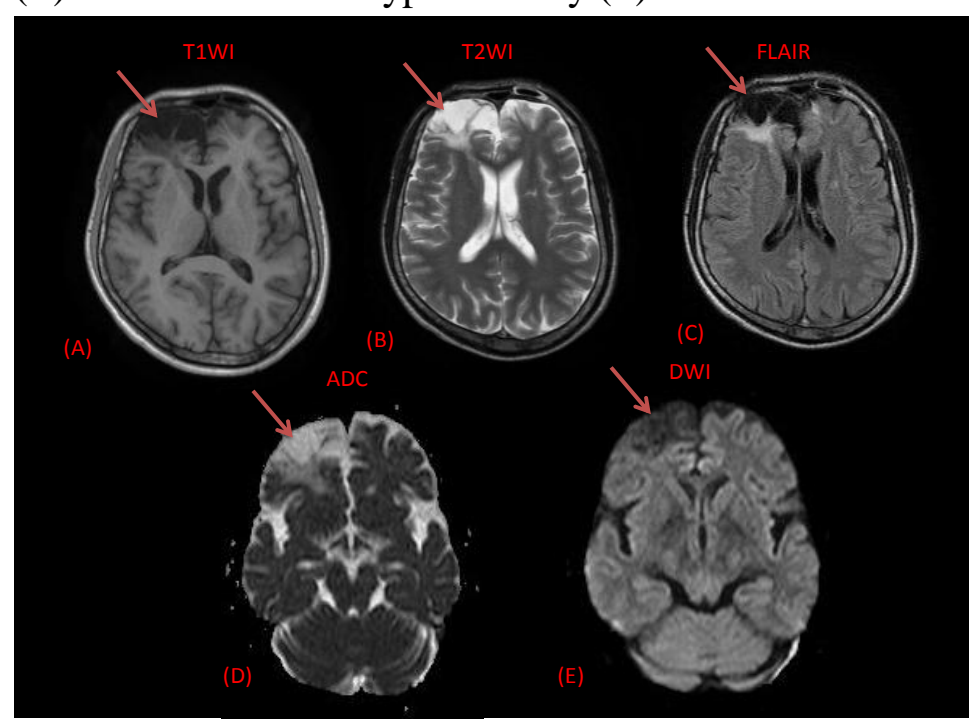

Figure 2: MRI images of a chronic right MCA territory infarct. T1WI axial sequence shows hypointense area in the right frontal lobe and in the parafalcine region of left frontal lobe (A), T2WI axial sequence shows hyperintensity (B) FLAIR sequences shows hypointensity (C), No diffusion restriction is noted in the DWI sequence (E) and ADC shows hyperintensity (D)

Table 1: Distribution of Age

\begin{tabular}{|c|c|c|}
\hline Age (range in years) & Frequency & Percent \\
\hline $\mathbf{3 1}-\mathbf{4 0}$ & 2 & $4.0 \%$ \\
\hline $\mathbf{4 1}-\mathbf{5 0}$ & 6 & $12.0 \%$ \\
\hline $\mathbf{5 1}-\mathbf{6 0}$ & 19 & $38.0 \%$ \\
\hline $\mathbf{6 1}-\mathbf{7 0}$ & 23 & $46.0 \%$ \\
\hline Total & 50 & $100.0 \%$ \\
\hline
\end{tabular}

The age of the patients with intra cranial lesions studied ranged from 30 years to 70 years with a mean age of $51.97 \pm 12.50$ years. More number of patients belongs the age group 61-70 years (46.0\%) followed by the age group of 51-60 years (38.0\%) followed by the groups $41-50$ years $(12.0 \%)$ and $31-40$ years $(4.0 \%)$ respectively.

Graph 1: Distribution of Age

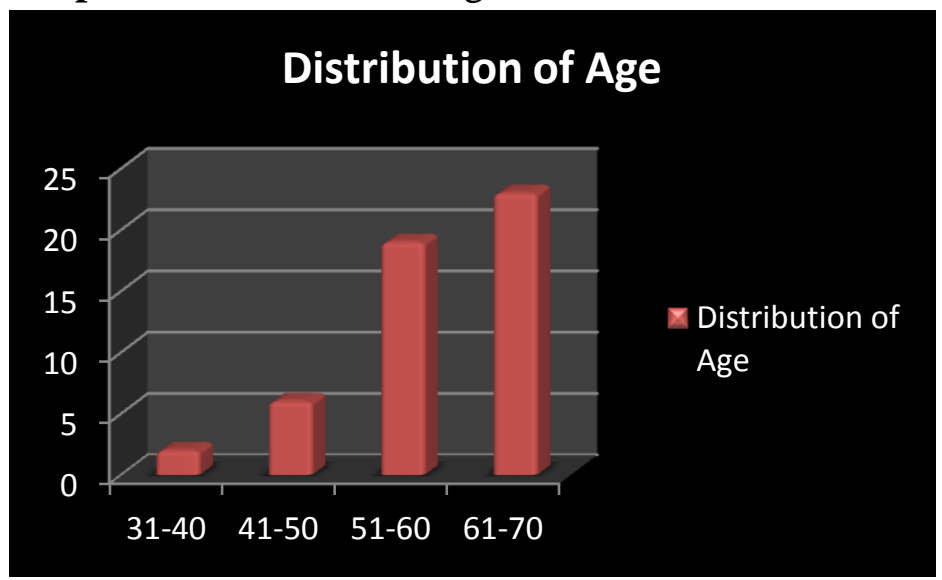

Table 2: Distribution of Diagnosis with Age

\begin{tabular}{|c|c|c|c|c|c|}
\hline \multirow{2}{*}{ Diagnosis } & \multicolumn{4}{|c|}{ Age Group } & \multirow{2}{*}{ Total } \\
\cline { 2 - 5 } & $\mathbf{3 1}-\mathbf{4 0}$ & $\mathbf{4 1}-\mathbf{5 0}$ & $\mathbf{5 1} \mathbf{- 6 0}$ & $\mathbf{6 1} \mathbf{- 7 0}$ & \\
\hline Acute Infarct & 2 & 6 & 14 & 14 & 36 \\
\hline $\begin{array}{c}\text { Chronic } \\
\text { Infarct }\end{array}$ & 0 & 0 & 3 & 8 & 11 \\
\hline $\begin{array}{c}\text { Sub-acute } \\
\text { Infarct }\end{array}$ & 0 & 0 & 2 & 1 & 3 \\
\hline Total & 2 & 6 & 19 & 23 & 50 \\
\hline
\end{tabular}

Arterial ischemic Infarct was seen in all age groups. However the peak $(46.0 \%)$ was noted in the group 61-70 years followed by the groups 51$60(38.0 \%)$ years, $41-50$ years $(12.0 \%)$ and $31-40$ years $(4.0 \%)$. 


\section{JMSCR Vol||05||Issue ||03||Page 18450-18456||March}

Graph 2: Distribution of Diagnosis with Age

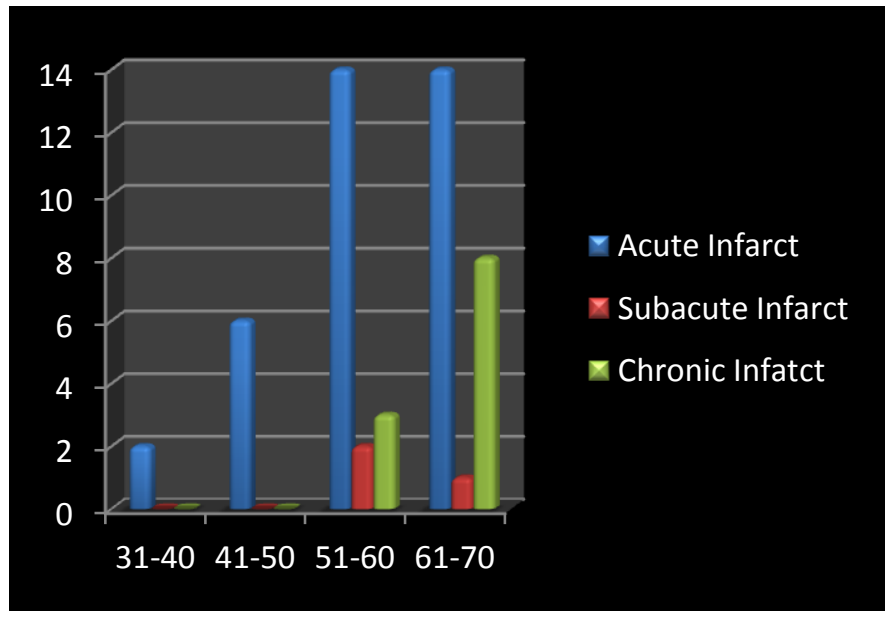

Table 3: Distribution of Sex

\begin{tabular}{|l|c|c|}
\hline & Frequency & Percent \\
\hline Male & 31 & $62.0 \%$ \\
\hline Female & 19 & $38.0 \%$ \\
\hline Total & 50 & $100.0 \%$ \\
\hline
\end{tabular}

Of the 50 patients studied, $62.0 \%$ were males and $38 \%$ were females. The mean age among males was $52.36 \pm 10.72$ years and the mean age among females was $51.36 \pm 15.01$ years.

Graph 3: Distribution of Sex

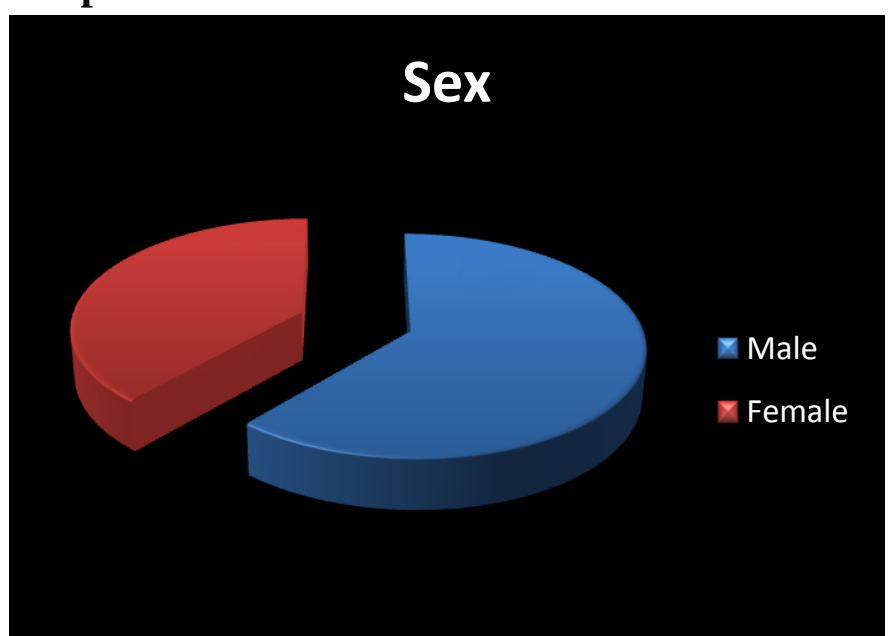

Imaging characteristics of various Arterial ischemia.

Of the 50 patients included in this study, 36 cases (72\%) showed hyperintensity on DWI. 38 cases (76\%) showed hypointensity on ADC images. All of these were hyperintenseon DW images and 3 cases of subacute infarct.
Table 4: Distribution of DWI

\begin{tabular}{|l|c|c|}
\hline & Frequency & Percent \\
\hline ISO & 3 & $6.0 \%$ \\
\hline HYPO & 11 & $22.0 \%$ \\
\hline HYPER & 36 & $72.0 \%$ \\
\hline Total & 50 & $100.0 \%$ \\
\hline
\end{tabular}

Graph 4: Distribution of DWI

\section{Distribution of DWI}

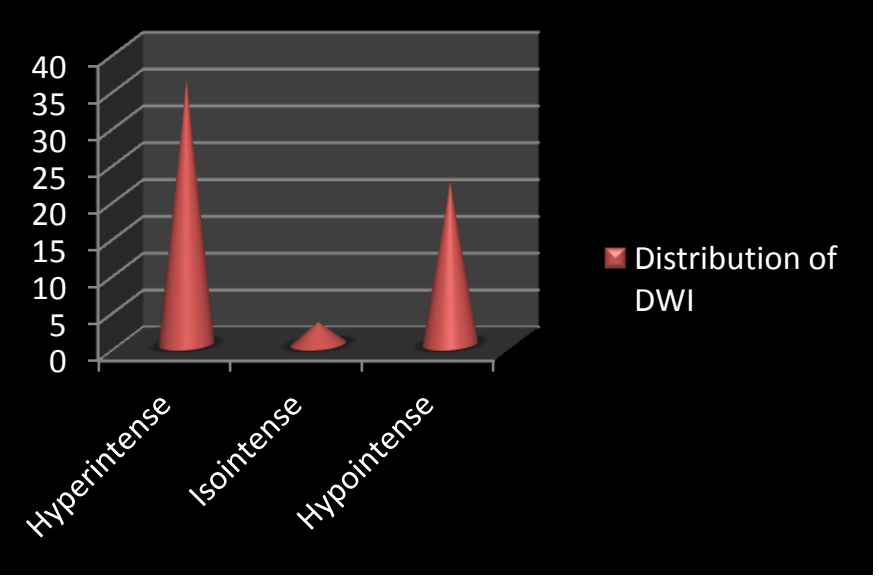

Table 5: Distribution of ADC

\begin{tabular}{|l|c|c|}
\hline & Frequency & Percent \\
\hline ISO & 1 & $2.0 \%$ \\
\hline HYPO & 38 & $76.0 \%$ \\
\hline HYPER & 11 & $22.0 \%$ \\
\hline Total & 50 & $100.0 \%$ \\
\hline
\end{tabular}

Graph 5: Distribution of ADC

\section{Distribution of ADC}

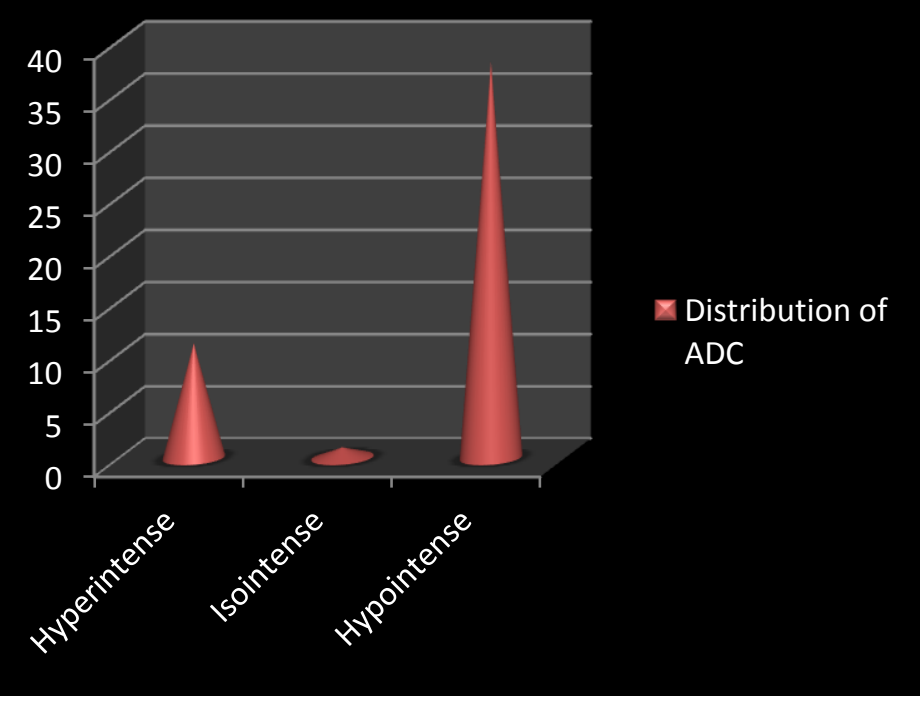


Table 6: Distribution of T2 Flair

\begin{tabular}{|l|c|c|}
\hline & Frequency & Percent \\
\hline ISO & 1 & $2.0 \%$ \\
\hline HYPO & 11 & $22.0 \%$ \\
\hline HYPER & 38 & $76.0 \%$ \\
\hline Total & 50 & $100.0 \%$ \\
\hline
\end{tabular}

Graph 6: Distribution of T2 Flair

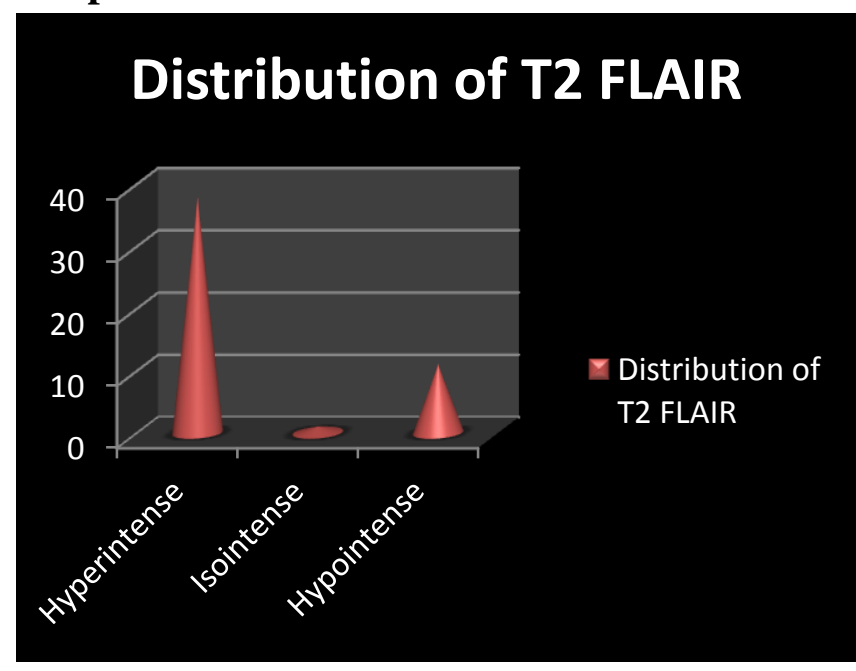

Infarcts

Table 7: Distribution of Infarcts according to age

\begin{tabular}{|l|l|l|}
\hline & Frequency & Percent \\
\hline Acute Infarct & 36 & $72.0 \%$ \\
\hline Chronic Infarct & 11 & $22.0 \%$ \\
\hline Sub-acute Infarct & 3 & $6.0 \%$ \\
\hline Total & 50 & $100.0 \%$ \\
\hline
\end{tabular}

Infarcts constituted $50.0 \%$ of cases of the total cases in this study. Of this, $72.0 \%$ were acute infarcts, $22.0 \%$ were chronic infarcts and $6.0 \%$ were sub-acute infarcts. The age of the patients with infarcts ranged from 35 to 71 years with a mean age of $58.24 \pm 8.99$ years. There were 30 $(60.0 \%)$ males and 20 (40.0\%) females among these cases.
Graph 7: Distribution of Infarcts according to the age

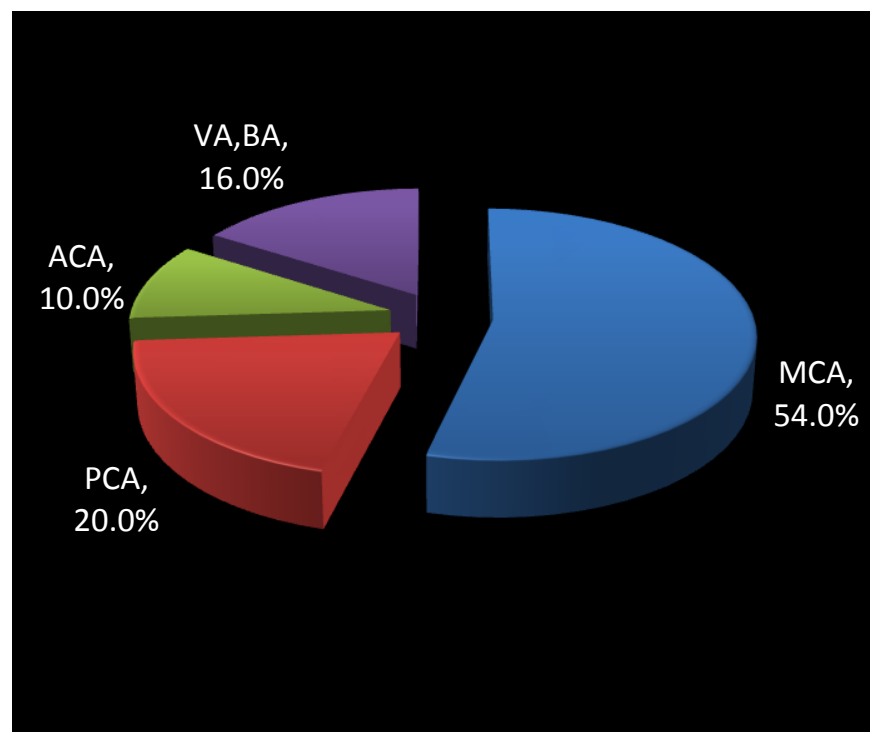

Graph 8: Distribution of Infarcts based on vascular territory:

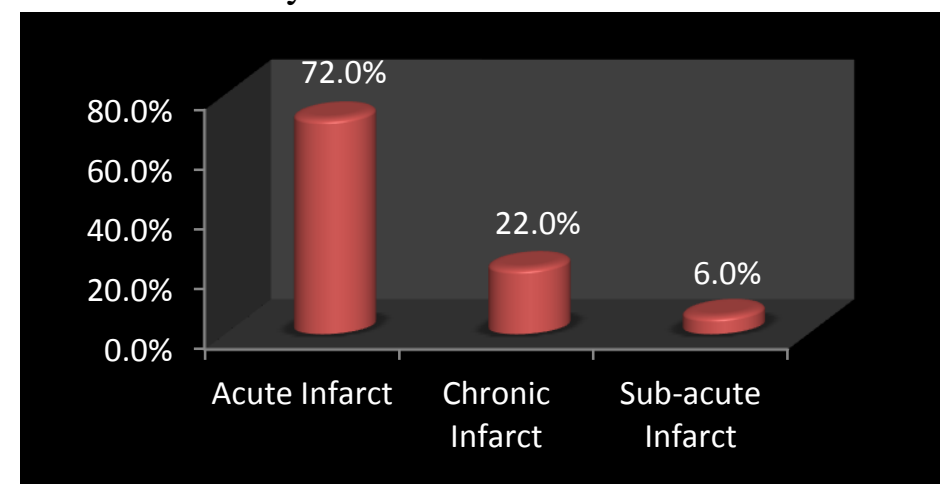

In 27 cases $(54 \%)$ the infarcts were in MCA territory, in 5 cases $(10 \%)$ they were in ACA territory, in 10 cases $(20 \%)$ the infarcts were in PCA territory and in 8 cases (16\%) they were in basilar artery and vertebral artery territory.

All 36 cases $(100 \%)$ of acute infarcts showed true diffusion restriction with hyperintensity on DWI and hypointensity on ADC images. Of these, 31 cases $(86.1 \%)$ showed hyperintensity on $\mathrm{T} 2 \mathrm{~W}$ images. The remaining 5 cases (13.8\%) showed no signal change on $\mathrm{T} 2 \mathrm{~W}$ images.

Of the 11 cases of chronic infarcts, ADC signal was increased in all, suggesting increased water diffusivity. In 5cases (45.5\%), there was hypointensity on DWI and T2 FLAIR images with hyperintensity on ADC images indicating encephalomalacic changes. T2 shine through was 
noted in 6 cases $(54.5 \%)$. None of the cases showed T2 washout.

Out of 3 cases of subacute infarcts, 1 (33.3\%) showed true restriction and $2(66.7 \%)$ showed T2 shine through.

\section{DISCUSSION}

Diffusion weighted MRI provides image contrast that is different from that provided byconventional MRI sequences. It provides a technique for mapping proton contrast thatreflects the microvascular environment. This imaging technique is sensitive to earlyischemic insult. DWI is performed with a pulse sequence capable of measuring water translation over short distances. This water diffusion is much slower in certainpathological conditions as compared with normal brain. ${ }^{(4)}$ In this study 50 patients with suspected stroke detected on DW MRI of the brain were included.

\section{Infarcts}

The sensitivity and specificity of DWI in the detection of acute ischemia is $100 \%$. The difference in sensitivity of DWI and conventional MRI sequences is more in the initial time period and decreases as time progresses. Results of this study are correlated with a study done by Gonzalez et al ${ }^{(2)}$ who concluded that DWI is superior to conventional MRI in the diagnosis and characterization of acute infarct.

In this study restricted diffusion was noted in $100 \%$ of acute infarcts. In $13.8 \%$ of acuteinfarcts, no change was noted on T2WI. Thus DWI was noted to be superior to T2WI in detection of acute infarcts.

In subacute infarcts and chronic infarcts, abnormal signal was noted on T2WI and on DWI in all patients. Thus there was no difference in their sensitivity for later stages of infarcts.

Rima K et al (4) showed that restricted diffusion is present in all patients on DW

MR studies obtained within 24 hours of the onset of symptoms, and in $94 \%$ of patients scanned after 2 weeks. In this study sub acute infarcts were defined as patients in whom imaging was performed between 2 and 14 days after symptom onset. $^{(3)}$

True diffusion restriction was noted in $33.3 \%$ of patients with subacute infarcts. The other $66.7 \%$ showed $\mathrm{T} 2$ shine through.

In this study $54 \%$ of infarcts were noted to be in MCA territory, $20 \%$ in PCA territory,

$10 \%$ in ACA territory and $16 \%$ in vertebral artery and basilar artery territory. This is comparable to a study done by Van Der Zwan et al ${ }^{(5)}$ which showed that MCA territory is the most common site for infarcts and ACA territory is the least commonamong major arterial territories.

In chronic infarcts the signal on DWI and ADC images is variable and depends on acombination of T2 signal and increased ADC values. The T2 signal is also affected by theonset of cystic encephalomalacia. (1) In this study T2 shine through was noted in $54.5 \%$ of chronic infarcts.

\section{CONCLUSION}

Diffusion weighted MRI is a valuable technique that provides unique information about the physiological state of brain tissue. The current study comprised 50 patients evaluated in Sri Manakula Vinayagar Medical College and Hospital, Puducherry who underwent DW MRI of the brain when they were referred for suspected stroke. All the MRI scans in this study were performed using 1.5T MRI scanner (Philips).

By using a combination of various MR sequences coupled with DWI and ADC images a valuable diagnosis may be provided to the clinicians. In this study the signal characteristics of various lesions on DWI, ADC, T2-FLAIR were studied.

Diffusion weighted MRI has been proven to be of excellent use in the characterization of infarcts and in the detection of acute infarcts. It is especially useful in the initial few hours of the ischemic insult when conventional MR sequences may be inconclusive and may not detect the infarct. In the setting of multiple infarcts it helps detect the acute ones and is helpful in differentiating acute, sub-acute and chronic infarcts. Thus diffusion weighted MR imaging has 
to be included in any standard imaging protocol for stroke patients.

\section{SUMMARY}

The objectives of this study were to describe the features of intracranial lesions on DWI and to compare these features with ADC and T2 FLAIR images. DW MRI Study was undertaken in 50 patients admitted to Sri ManakulaVinayagar Medical College and Hospital, Puducherry with suspected stroke.

All the MRI scans in this study were performed using 1.5 T MRI scanner (Philips). The study comprised $61 \%$ males and $39 \%$ females. Infarcts constituted $100.0 \%$ of cases of the total cases in this study. Of this, $72.0 \%$ were acute infarcts, $22.0 \%$ were chronic infarcts and $6.0 \%$ were subacute infarcts.

All cases of acute infarcts and $33 \%$ of sub-acute infarcts showed diffusion restriction. None of the chronic infarcts showed true restriction of diffusion. No signal abnormality was noted in $13.8 \%$ of acute infarcts on $\mathrm{T} 2 \mathrm{~W}$ images. Thus DWI not only helped detect all cases of acute infarcts but also proved to be a useful tool in categorizing the infarcts as acute, chronic and subacute infarcts.

\section{REFERENCES}

1. Schaefer PW, Grant PE, Gonzalez RG. Diffusion weighted MR imaging of thebrain. Radiology 2000 november; 217:331-345.

2. Gonzalez RG, Schaefer PW, Buonanno FS, et al. Diffusion-weighted MRimaging: diagnostic accuracy in patients imaged within 6 hours of strokesymptom onset. Radiology 1999;210:155-162.

3. Osborn AG, Salzman KL, Barkovich AJ, Katzman GL, Provenzale JM,

4. Hansberger HR et al. Diagnostic imaging brain. 2nd ed. Canada: Amirsys; 2010.

5. K Rima, G Rohit, P Anjali, C Veena. Role of diffusion weighted MR imaging inearly diagnosis of cerebral infarction. Ind $\mathrm{J}$ RadiolImag 2003;3(2):213-217.

6. Van der Zwan A, Hillen B, Tulleken $\mathrm{H}$ et al. variability of the major cerebralarteries. J Neurosurg 1992;77:927-940. 